\title{
Cycling@CHI: Towards a Research Agenda for HCI in the Bike Lane
}

GIAN-LUCA SAVINO, University of Bremen, Germany

TAMARA VON SAWITZKY, Technische Hochschule Ingolstadt, Germany

ANDRII MATVIIENKO, Technical University of Darmstadt, Germany

MIRIAM STURDEE, Lancaster University, United Kingdom

PAWEŁ W. WOŹNIAK, Utrecht University, the Netherlands

MARKUS LÖCHTEFELD, Aalborg University, Denmark

ANDREW L. KUN, University of New Hampshire, United States

ANDREAS RIENER, Technische Hochschule Ingolstadt, Germany

JONNA HÄKKILÄ, University of Lapland, Finland

In this workshop, we will explore and discuss future developments in mobile user-interfaces for cyclists and users of similar interfaces or services. We highlight the challenge of balancing safety and ecological validity in experiments, and how novel and improved evaluation methods can improve the current situation. We aim to bring together researchers with a strong background in designing and evaluating novel user interfaces in the domain of bicycles and mobility, as well as practitioners who build consumer products in that domain. The workshop's goal is to explore novel ways of designing and evaluating user interfaces for cyclists and similar users when it comes to interacting with mobile devices and services on the ride.

CCS Concepts: • Human-centered computing $\rightarrow$ Interaction techniques; User interface design; HCI design and evaluation methods; Virtual reality; Mixed/augmented reality.

Additional Key Words and Phrases: cycling, mobile interaction, user interface, evaluation

\section{ACM Reference Format:}

Gian-Luca Savino, Tamara von Sawitzky, Andrii Matviienko, Miriam Sturdee, Paweł W. Woźniak, Markus Löchtefeld, Andrew L. Kun, Andreas Riener, and Jonna Häkkilä. 2021. Cycling@CHI: Towards a Research Agenda for HCI in the Bike Lane. In CHI Conference on Human Factors in Computing Systems Extended Abstracts (CHI '21 Extended Abstracts), May 8-13, 2021, Yokohama, Japan. ACM, New York, NY, USA, 8 pages. https://doi.org/10.1145/3411763.3441316

\section{BACKGROUND}

Bicycles are becoming an increasingly important means of transport in many cities as they complement or replace the car or public transport as a viable option. In Germany alone, the number of e-bikes sold doubled in the last five years ${ }^{1}$. While there are many reasons for that, e.g., healthier lifestyles, especially in 2020, we also saw a general increase in bicycle sales worldwide due to the COVID-19 pandemic. For example, in April 2020, 60\% more bicycles were sold in

\footnotetext{
${ }^{1}$ https://de.statista.com/statistik/daten/studie/152721/umfrage/absatz-von-e-bikes-in-deutschland/
}

Permission to make digital or hard copies of part or all of this work for personal or classroom use is granted without fee provided that copies are not made or distributed for profit or commercial advantage and that copies bear this notice and the full citation on the first page. Copyrights for third-party components of this work must be honored. For all other uses, contact the owner/author(s).

(c) 2021 Copyright held by the owner/author(s).

Manuscript submitted to ACM 

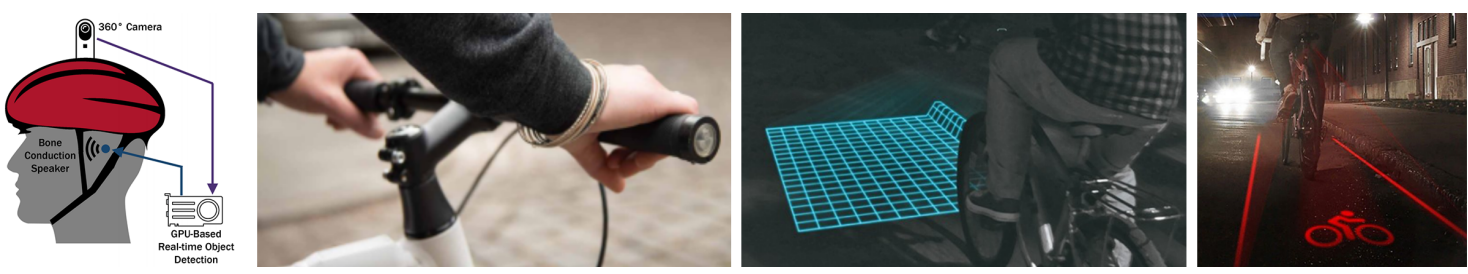

Fig. 1. Examples of existing technologies to support cyclists using augmentations to helmets, vibration bicycle grips, and projected surfaces, as discussed in [8].

the United Kingdom compared to $2019^{2}$. At the same time, we see more and more cities adding e-scooters [21] to complement public transport and bike-sharing services to give even more people access to two-wheeled transportation.

These developments have led to a rapid increase in (non-)motorized vulnerable road users (VRUs) using these kinds of transportation methods. As this happens - and especially as cycling becomes one of the main transportation methods for daily commutes, more people will start interacting with mobile devices and connected services (e.g., navigation applications) while riding. Studies in the past have shown that, even though this can be dangerous [2], people interact with their mobile phones while in motion, including cycling [7]. Additionally, there is a worrying result which accompanies this development: According to a recent report of the European Transport Safety Council [3], the numbers of bicycle accidents stagnated in the last decade, while the numbers decreased for other forms of traffic. Based on these insights, we expect to see similar developments for other road users in bike lanes using, for example, e-scooters or e-bikes.

To account for this problem, researchers have proposed a wide variety of novel bike interaction methods, including tactile [9], audio [11], wearable, projection based [10], and gesture based interfaces [12], among others (Figure 1). Examples include Tacticycle by Pielot et al. [16], where vibrating handlebars indicate the direction in which the destination lies in an as-the-crow-flies manner. Dancu et al. [1] presented Gesture Bike, which, through hand gestures, allowed cyclists to control a turn indicator mounted to the bicycle. Finally, Woźniak et al. [25] created custom handlebar controllers to enable the user to interact with the most common smartphone features like accepting a phone call or playing/pausing music. These systems do not only enable cyclists to interact with information while cycling. Most of them also aim towards making this interaction as safe as possible by directing the user's visual attention away from the mobile device. Unfortunately, very few, if any, of these implementations or results have made it into consumer products yet. Consequently, long-existing and newly developed bike computers and navigation software still use an interface design language known from the car domain without much adaptation to cyclists' needs, making use of touch-screens attached to the handlebar.

In addition to the developments in interface design, increasingly popular tracking and monitoring apps also create new possibilities, especially for cyclists. It has been found that self-monitoring is most relevant for performanceoriented competitive or lifestyle cyclists, and the interesting features are often simple measures such as speed, time, and distance [17]. Moreover, tracking apps and networked bicycles can create big data as part of the smart cities [18]. Cyclists can be harnessed as a data source, for instance, used for the smart cities for urban and route planning [14]. They could also benefit from smart city/traffic technologies that communicate possible dangers between traffic participants, allowing cyclists to know about possible imminent dangers ahead of time, which could prevent accidents [23]. The possibilities to use tracking data for novel features for cyclists and bike-integrated UIs are still under-explored. Also,

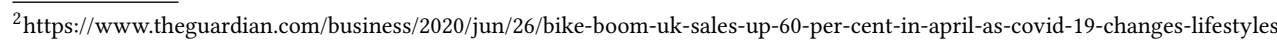



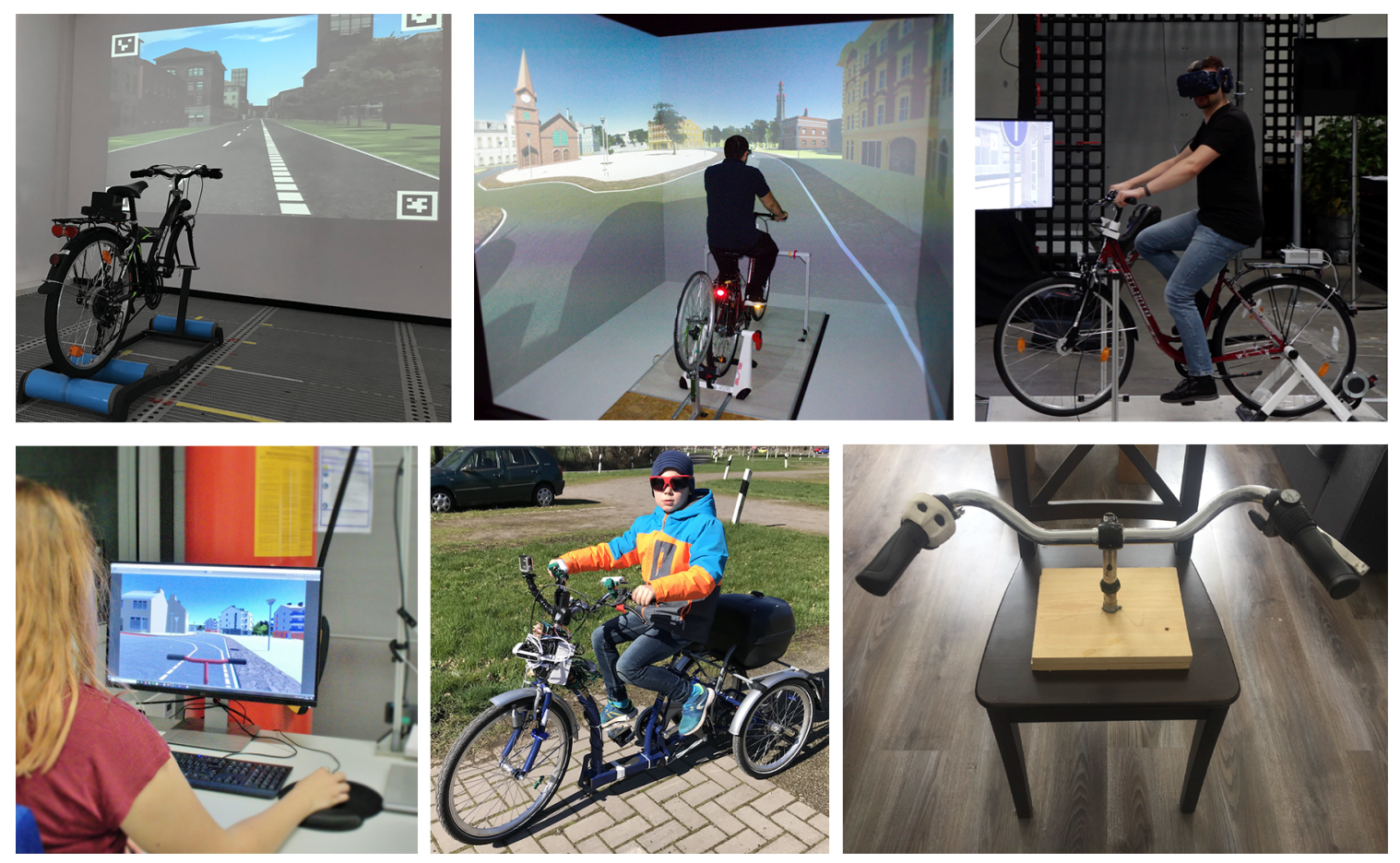

Fig. 2. Examples of evaluation methods showing different simulator setups in the lab, a tricycle on the outdoor test-track, and a prototype mounted on handlebars as discussed in [9-12, 22, 24, 25].

the possibilities for HCI in outdoor recreational activities have lately gained interest [4], and the UI design solutions optimized for cycling in urban and outback contexts may have different requirements.

Designing and evaluating novel technologies, services, and methods for sports [13] and outdoor activities [5] have in the past often been a focus at $\mathrm{CHI}$. With this workshop, we want to start building a well-defined community in $\mathrm{CHI}$ to develop a future research agenda for interactive devices for cycling.

\subsection{Issues to be Addressed}

As more road users move to the bike lane, we are less dealing with cyclists' issues specifically but with more general issues concerning interaction on the ride, one-handed interaction, cognitive load, and safety, among others. One of the issues to tackle in this workshop is understanding the development of bicycle- and similar user interfaces and services, their implications for future designs, and how to cater for different road users effectively.

Another major issue in this domain, which could partly be responsible for the slow adoption rate of new systems, is delivering generalizable results with high ecological validity. Often experiments include only one or very few simple tasks, only one route, are done on a dedicated test-track or in the lab (Figure 2). The main reason behind these decisions is to keep the participants safe. Evaluating novel interfaces in a real-world environment can be potentially dangerous for participants and other road users. Therefore, researchers usually have to make a trade-off between ecological validity and safety. The latest addition to already used evaluation methods is virtual reality (VR). Simulators which can be used in front of a screen or with head-mounted displays offer the potential to test novel interaction on the ride without 
safety concerns. One of the issues to tackle in this workshop is how to compare results from virtual or mixed reality simulation to real-world performance $[15,19]$. Additionally, VR also gives the possibility to explore novel or completely unrealistic settings or even deceive the user [6].

Lastly, the introduction of mobility infrastructures such as bike and e-scooter rental has not been without conflicts, e.g., due to the vehicles' problematic abandonment in urban spaces [21]. Thus, attention is needed to investigate the design elements essential for desirable user experiences (UX) not only for road users but also those surrounding them. Many of these vehicles come with a built-in smartphone-mount, whereas novel e-bikes like the VanMoof $3^{3}$ come with built-in buttons and displays. Looking at these developments, designers should consider the risks and potential consequences of introducing technology-enhanced biking in urban spaces.

\subsection{Goal of the Workshop}

The goal of this workshop is twofold. (1) Given the increasing number of riding VRUs, we want to prototype, discuss and explore novel interfaces and their social impact which work across transportation methods with similar constraints, like bicycles, e-bike, e-scooters, etc. (2) Further, we plan to address how to safely evaluate these systems by maintaining a high ecological validity that allows our research results to translate better to real-world environments. We will especially discuss the use of simulators and VR/MR methods. By this, we want to develop a shared understanding of mobile interaction on the ride and discuss how we can join efforts to work towards developing a promising future research agenda around $\mathrm{HCI}$ in the bike lane.

\section{ORGANIZERS}

Main contact person: Gian-Luca Savino

Gian-Luca Savino is a Ph.D. student at the Human-Computer Interaction Group at the University of Bremen. His focus is on novel bicycle navigation and interaction with mobile devices, in particular for as-the-crow-flies navigation. Tamara von Sawitzky is a Ph.D. student at Johannes Kepler University Linz and a researcher at Technische Hochschule Ingolstadt. Her research focuses on HUD-based assistance systems for cyclists and their evaluation in VR/MR simulation. Andrii Matviienko is a postdoctoral researcher at Technical University of Darmstadt. His research focus lies on designing assistance systems for cyclists, educational technology for children and people with special needs. He is also a co-organizer of the SIGCHI sponsored International HCI Summer School on Cycling 4 .

Miriam Sturdee is a research fellow at Lancaster University, UK. Her work focuses on creative practices within computer science, with an emphasis on visual methods and the design of novel interfaces.

Paweł W. Woźniak is an Assistant Professor at Utrecht University. He focuses on understanding the design of technologies that enhance our wellbeing and experience of physical activity.

Markus Löchtefeld is an Associate Professor at Aalborg University. His research focuses on wearable and tangible computing as well as novel prototyping techniques.

Andrew L. Kun is professor of electrical and computer engineering at the University of New Hampshire. His research focuses on in-vehicle user interfaces, and more broadly on technologies to support work and wellbeing.

Andreas Riener is professor for HCI and VR at Technische Hochschule Ingolstadt and director of the UXD programs. He conducts hypotheses-driven quasi-experimental research in human-computer interaction in the broader context of mobility.

\footnotetext{
${ }^{3}$ https://www.vanmoof.com/

${ }^{4}$ https://cycling.offis.de
} 
Jonna Häkkilä is a professor at Faculty of Art and Design, University of Lapland. She conducts research in the cross-section of technology and design in outdoor and nature contexts, and on mobile and wearable computing for sports and wellness.

\section{WEBSITE}

A website will be set up to inform participants about the workshop and make potential position papers available to them.

\section{PRE-WORKSHOP PLANS}

The workshop will bring together participants from both academia and industry. We will announce the Call for Participation at popular mailing lists, calendars, and social media (e.g., Twitter, Facebook). Furthermore, we will directly contact researchers working in the fields of bicycle interaction, user interfaces, mobility, and related areas. Since we are aiming for a strong collaboration with industry practitioners, we will reach out to leading players in the mobile navigation, cycling accessories, and bike-sharing community. We will continuously promote the workshop during the period leading up to the workshop deadline.

A workshop website will be set up to provide information about the workshop topics and offer relevant material to support people interested in the topic. Accepted submissions will be made available through the website before the start of the workshop.

\section{WORKSHOP STRUCTURE}

The workshop runs across one full day. It will consist of workshop paper position pitches, hands-on exercises, and discussions. The estimated number of workshop participants is around 25-30 and should be a good mix of academic researchers and industry practitioners. Each participant contributes a position paper which presents the participants' interest and research area as well as their focus of contribution they want to bring to the workshop. The workshop committee will review position papers, and participants will be selected based on the novelty, inspirational aspect, and fit for the workshop. Based on the position papers' topics, the practical sessions of the workshop will be further refined. To stimulate discussion, we will conduct a design fiction workshop in which participants will develop concepts of future bicycle interfaces. This will help us identify key future challenges for HCI in the bike lane.

\subsection{Preliminary Workshop Schedule}

- Introduction and position pitches

- Presentation of the two hands-on sessions and discussion

- Coffee break

- Interactive session 1: Design Fiction

- Lunch

- Interactive session 2: Evaluation

- General discussion on future directions

- Wrap-up

Each interactive session will start with a short presentation/demonstration by one of the committee members. Before the workshop there will be a Cycling@Home session. 


\subsection{Cycling@Home Session}

In order to facilitate creative ideas for the workshop we will organise a pre-workshop cycling at home event. We will ask participants to take their bike out for a ride wherever they live. Participants can choose to imagine novel interfaces and how they would use them, they could consciously experience constraints while riding or report about special circumstances that potentially differentiate their cycling experience from others. For participants who can't go on a tour themselves we are planning to organise remote-cycling where those people are taken on a live-video tour with someone else. This event will of course be completely voluntary and will happen the week before the workshop. We will prepare some guidelines and examples to post on our workshop website.

\subsection{Interactive Session 1: Design Fictions}

Participants will be split into groups of four to five people to create design fictions of future bicycle interfaces. This session will incorporate ideation and design fiction techniques to engage participants in "world-building" and storytelling for their prototypes, using visual narrative and sketching to support their idea formation [20]. After 50 minutes, each group will have 10 minutes to present their prototype(s) and imagined worlds before the lunch break (Presentations).

\subsection{Interactive Session 2: Evaluation}

In the second interactive session, we would like to focus on the evaluation methods and environments when designing technology for cyclists and other VRUs. As a starting point, we will use the prototypes developed in the first session and ask a different group to develop a potential evaluation for it, aiming to maintain a high ecological validity of the experiments and participants' safety. Here, we also aim to include aspects that can impact the measures depending on the possible experiment environments (VR, MR, outdoor, etc.). After 50 minutes, each group will have 10 minutes to present their evaluation method.

\subsection{Tools for Communication and Collaboration}

Due to the current circumstances we are preparing the workshop to work as a virtual workshop. Through past conferences we have collected experiences with various resources and will facilitate them to guarantee a great virtual workshop experience for everyone. We have first hand experience and will facilitate some of the following tools (among others), depending on the size of the workshop:

- Miro as online collaborative whiteboards

- Zoom breakout rooms for group discussions and live prototyping

- Remo for collaborative group work

- Slack to communicate with the workshop participants before, during and after the workshop

\section{POST-WORKSHOP PLANS}

This workshop's primary goal is to bring together people from different areas to discuss how to develop assistive technology for cyclists and similar riding VRUs. We plan to make the workshop's outcomes accessible for the research community by keeping the accepted position papers on the workshop's website. Additionally, we will summarise the presented works, discussions, and results of the workshop via a magazine article (e.g., ACM Interactions). In this article, we will define future design spaces and opportunities for future research. As the research area of assisting technology for cyclists is underrepresented in the HCI research community, we will also ask workshop participants to submit an 
extended article of their submission for a special journal issue (e.g., in Personal Ubiquitous Computing) that we aim to organize. This information and call for papers will also be shared on the website and will be open to other interested researchers in the community.

\section{CALL FOR PARTICIPATION}

Bicycles are becoming an increasingly important means of transport in many cities as they complement or replace the car or public transport. Besides increasing numbers of cyclists, we see new forms of transportation (e.g., e-scooters) sharing not only interaction-related aspects with them but also the bike lane. As this development continues, safe mobile interaction on the ride through suitable and effective user interfaces is needed. In the past, researchers have proposed a variety of novel interaction methods. Many of which have not made it into consumer products yet. One reason is the difficulty of testing such systems with real users in real environments. Issues like safety, generalisability, and ecological validity often have to be carefully balanced.

This workshop focuses on designing and evaluating novel user interfaces for interaction on the ride through

(1) Prototyping and discussing novel interfaces and services for the bike lane

(2) Finding ways to safely evaluate these systems while maintaining high ecological validity

We invite authors to submit a position paper of up to 5 pages (new ACM single-column format) showcasing their interest or experience in the topic. Position papers in PDF-format are due by February 14, 2021. Admittance will be based on workshop relevance and the potential of contributing to discussions and actions, as reviewed by the workshop organisers. At least one author must attend the workshop. All participants must register for the workshop and at least one day of the conference. For more detailed information, visit the workshop's website: https://cyclingatchi.wordpress.com/.

\section{REFERENCES}

[1] Alexandru Dancu, Velko Vechev, Adviye Ayça Ünlüer, Simon Nilson, Oscar Nygren, Simon Eliasson, Jean-Elie Barjonet, Joe Marshall, and Morten Fjeld. 2015. Gesture Bike: Examining Projection Surfaces and Turn Signal Systems for Urban Cycling. In Proceedings of the 2015 International Conference on Interactive Tabletops \& Surfaces (Madeira, Portugal) (ITS '15). Association for Computing Machinery, New York, NY, USA, $151-159$. https://doi.org/10.1145/2817721.2817748

[2] Dick de Waard, Paul Schepers, Wieke Ormel, and Karel Brookhuis. 2010. Mobile phone use while cycling: Incidence and effects on behaviour and safety. Ergonomics 53, 1 (2010), 30-42. https://doi.org/10.1080/00140130903381180 PMID: 20069479.

[3] European Transport Safety Council. 2020. HOW SAFE IS WALKING AND CYCLING IN EUROPE? PIN Flash Report 38. (2020). https://etsc.eu/wpcontent/uploads/PIN-Flash-38_FINAL.pdf

[4] Michael D Jones, Zann Anderson, Jonna Häkkilä, Keith Cheverst, and Florian Daiber. 2018. HCI outdoors: understanding human-computer interaction in outdoor recreation. In Extended Abstracts of the 2018 CHI Conference on Human Factors in Computing Systems. 1-8.

[5] Michael D. Jones, Zann Anderson, Jonna Häkkilä, Keith Cheverst, and Florian Daiber. 2018. HCI Outdoors: Understanding Human-Computer Interaction in Outdoor Recreation. In Extended Abstracts of the 2018 CHI Conference on Human Factors in Computing Systems (Montreal QC, Canada) (CHI EA '18). Association for Computing Machinery, New York, NY, USA, 1-8. https://doi.org/10.1145/3170427.3170624

[6] Markus Löchtefeld, Antonio Krüger, and Hans Gellersen. 2016. DeceptiBike: Assessing the Perception of Speed Deception in a Virtual Reality Training Bike System. In Proceedings of the 9th Nordic Conference on Human-Computer Interaction (Gothenburg, Sweden) (NordiCHI '16). Association for Computing Machinery, New York, NY, USA, Article 40, 10 pages. https://doi.org/10.1145/2971485.2971513

[7] Joe Marshall, Alexandru Dancu, and Florian "Floyd" Mueller. 2016. Interaction in Motion: Designing Truly Mobile Interaction. In Proceedings of the 2016 ACM Conference on Designing Interactive Systems (Brisbane, QLD, Australia) (DIS '16). Association for Computing Machinery, New York, NY, USA, 215-228. https://doi.org/10.1145/2901790.2901844

[8] Andrii Matviienko. 2020. Designing Multimodal Assistance Systems for Child Cyclists. OlWIR Verlag für Wirtschaft, Informatik und Recht.

[9] Andrii Matviienko, Swamy Ananthanarayan, Shadan Sadeghian Borojeni, Yannick Feld, Wilko Heuten, and Susanne Boll. 2018. Augmenting Bicycles and Helmets with Multimodal Warnings for Children. In Proceedings of the 20th International Conference on Human-Computer Interaction with Mobile Devices and Services (Barcelona, Spain) (MobileHCI '18). Association for Computing Machinery, New York, NY, USA, Article 15, 13 pages. https://doi.org/10.1145/3229434.3229479 
[10] Andrii Matviienko, Swamy Ananthanarayan, Stephen Brewster, Wilko Heuten, and Susanne Boll. 2019. Comparing Unimodal Lane Keeping Cues for Child Cyclists. In Proceedings of the 18th International Conference on Mobile and Ubiquitous Multimedia (Pisa, Italy) (MUM '19). Association for Computing Machinery, New York, NY, USA, Article 14, 11 pages. https://doi.org/10.1145/3365610.3365632

[11] Andrii Matviienko, Swamy Ananthanarayan, Abdallah El Ali, Wilko Heuten, and Susanne Boll. 2019. NaviBike: Comparing Unimodal Navigation Cues for Child Cyclists. In Proceedings of the 2019 CHI Conference on Human Factors in Computing Systems (Glasgow, Scotland Uk) (CHI '19). Association for Computing Machinery, New York, NY, USA, 1-12. https://doi.org/10.1145/3290605.3300850

[12] Andrii Matviienko, Swamy Ananthanarayan, Raphael Kappes, Wilko Heuten, and Susanne Boll. 2020. Reminding Child Cyclists about Safety Gestures. In Proceedings of the 9TH ACM International Symposium on Pervasive Displays (Manchester, United Kingdom) (PerDis '20). Association for Computing Machinery, New York, NY, USA, 1-7. https://doi.org/10.1145/3393712.3394120

[13] Florian "Floyd" Mueller, Joe Marshall, Rohit Ashok Khot, Stina Nylander, and Jakob Tholander. 2016. Jogging at CHI. In Proceedings of the 2016 CHI Conference Extended Abstracts on Human Factors in Computing Systems (San Jose, California, USA) (CHI EA '16). Association for Computing Machinery, New York, NY, USA, 1119-1122. https://doi.org/10.1145/2851581.2886435

[14] G Norman and N Kesha. 2015. Using smartphones for cycle planning. In IPENZ Transportation Group Conference. 22-24.

[15] Steve O'Hern, Jennie Oxley, and Mark Stevenson. 2017. Validation of a bicycle simulator for road safety research. Accident; analysis and prevention 100 (2017), 53-58. https://doi.org/10.1016/j.aap.2017.01.002

[16] Martin Pielot, Benjamin Poppinga, Wilko Heuten, and Susanne Boll. 2012. Tacticycle: Supporting Exploratory Bicycle Trips. In Proceedings of the 14th International Conference on Human-Computer Interaction with Mobile Devices and Services (San Francisco, California, USA) (MobileHCI '12). Association for Computing Machinery, New York, NY, USA, 369-378. https://doi.org/10.1145/2371574.2371631

[17] Lukasz Piwek, Adam Joinson, and James Morvan. 2015. The use of self-monitoring solutions amongst cyclists: An online survey and empirical study. Transportation Research Part A: Policy and Practice 77 (2015), 126-136.

[18] Gustavo Romanillos, Martin Zaltz Austwick, Dick Ettema, and Joost De Kruijf. 2016. Big data and cycling. Transport Reviews 36, 1 (2016), 114-133.

[19] Gian-Luca Savino, Niklas Emanuel, Steven Kowalzik, Felix Kroll, Marvin C. Lange, Matthis Laudan, Rieke Leder, Zhanhua Liang, Dayana Markhabayeva, Martin Schmeißer, Nicolai Schütz, Carolin Stellmacher, Zihe Xu, Kerstin Bub, Thorsten Kluss, Jaime Maldonado, Ernst Kruijff, and Johannes Schöning. 2019. Comparing Pedestrian Navigation Methods in Virtual Reality and Real Life. In 2019 International Conference on Multimodal Interaction (Suzhou, China) (ICMI '19). Association for Computing Machinery, New York, NY, USA, 16-25. https://doi.org/10.1145/3340555.3353741

[20] Miriam Sturdee, Paul Coulton, Joseph G Lindley, Mike Stead, Haider Ali, and Andy Hudson-Smith. 2016. Design fiction: How to build a Voight-Kampff machine. In Proceedings of the 2016 CHI Conference Extended Abstracts on Human Factors in Computing Systems. 375-386. https://doi.org/10.1145/ 2851581.2892574

[21] Sylvaine Tuncer and Barry Brown. 2020. E-scooters on the Ground: Lessons for Redesigning Urban Micro-Mobility. In Proceedings of the 2020 CHI Conference on Human Factors in Computing Systems. 1-14.

[22] Tamara von Sawitzky, Thomas Grauschopf, and Andreas Riener. 2020. The Next Stage of Road Traffic Education: A Mixed Reality Bicycle Simulator to Improve Cyclist Safety. In GI VR / AR Workshop, Benjamin Weyers, Christoph Lürig, and Daniel Zielasko (Eds.). Gesellschaft für Informatik e.V. https://doi.org/10.18420/vrar2020_31

[23] Tamara von Sawitzky, Thomas Grauschopf, and Andreas Riener. 2020. No Need to Slow Down! A Head-up Display Based Warning System for Cyclists for Safe Passage of Parked Vehicles. In 12th International Conference on Automotive User Interfaces and Interactive Vehicular Applications (Virtual Event, DC, USA) (AutomotiveUI '20). Association for Computing Machinery, New York, NY, USA, 1-3. https://doi.org/10.1145/3409251.3411708

[24] Tamara von Sawitzky, Philipp Wintersberger, Andreas Löcken, Anna-Katharina Frison, and Andreas Riener. 2020. Augmentation Concepts with HUDs for Cyclists to Improve Road Safety in Shared Spaces. In Extended Abstracts of the 2020 CHI Conference on Human Factors in Computing Systems (Honolulu, HI, USA) (CHI EA '20). Association for Computing Machinery, New York, NY, USA, 1-9. https://doi.org/10.1145/3334480.3383022

[25] Pawel W. Woźniak, Lex Dekker, Francisco Kiss, Ella Velner, Andrea Kuijt, and Stella F. Donker. 2020. Brotate and Tribike: Designing Smartphone Control for Cycling (MobileHCI '20). Association for Computing Machinery, New York, NY, USA. https://doi.org/10.1145/3379503.3405660 